\title{
Experience of peptic ulcer perforation over a decade in a teaching hospital of southern Bangladesh
}

\author{
Md Mizanur Rahuman ${ }^{1}$, Ashish Kumar Saha² and Abdur Rahim ${ }^{3}$
}

\begin{abstract}
Objective To determine disease characteristics, check the treatment status and compare outcome with other series.

Design and setting A retrospective study in a single unit of the Department of Surgery of Khulna Medical College Hospital, south west Bangladesh.

Patients and methods After diagnosis by clinical and radiological examination, 491 patients were treated during the period July 1992 to November 2002. Two options of treatment were carried out: simple closure and peritoneal lavage in 364 cases, and 127 patients managed by nonsurgical methods. Main outcome measures: mortality and morbidities.
\end{abstract}

Results Male: female ratio was $445: 46$, average age 43 years. Mortality in the surgical group was $6.8 \%$ and in the non-surgical group $0.02 \%$.

Conclusion Perforated peptic ulcer is prevalent even after the wide use of $\mathrm{H}_{2}$ receptor antagonists. Strict case selection for surgical and non-surgical treatment has reduced mortality. These results support the case for nonoperative treatment and for conservative surgery.

\section{Introduction}

Up to a couple of decades ago surgery for peptic ulcer led sugeons' operation lists. Today elective operations have reduced dramatically. But peptic ulcer perforation and emergency surgery is still prevalent. From the available published articles it is clear that presentation, epidemiology, disease characteristics and medical management have changed $(1,2)$. With the introduction of laparoscopy, management patterns have also changed more recently. The management of perforation is still a debatable issue even in the new millennium $(3,4)$. This study was carried out in Khulna Medical College Hospital located in a coastal area of Bangladesh from July 1992 to November 2002 to determine the age, sex and disease characteristics, mode of diagnosis, the status of treatment and its outcome.

\section{Patients and methods}

This retrospective study includes 491 patients with peptic ulcer perforation from July 1992 to November 2002. We analysed all the case records in detail. The condition of the patient was characterised as shock when systolic blood pressure was $<90 \mathrm{mmHg}$, pulse rate $>120$ beats per minute and the patient failed to respond to appropriate initial resuscitation.

The diagnosis of peptic ulcer perforation was usually made on the basis of a typical history and physical examination, supported by an erect chest xray showing a free subphrenic gas shadow, and ultrasonographic examination of the abdomen.

After admission every patient was provided with adequate resuscitation with intravenous fluids, nasogastric suction and application of broad spectrum antibiotics eg. amoxicillin+metronidazole or amoxicillin+gentamicin+metronidazole. Blood transfusion was needed in late cases or in patients with shock. Treatment was operative or nonoperative according to the following criteria.

Non-surgical: Fifty six and 71 patients were managed differently in two groups according to predetermined criteria. In the first group, patients were relatively healthy, presented early (within 12 hours of pain), and had minimum pain and tenderness over the abdomen. They were given intravenous fluids. Gastric aspiration was continued until

'Assistant Professor, ${ }^{2.3}$ Assistant Registrar, Department of Surgery, Khulna Medical College, Khulna 9000, Bangladesh. (Correspondence Md MR, email: mizansk7@khulna.bangla.net. Received 9 December 2002, accepted 18 April 2003). 
the abdomen became softer and bowel sounds were normal. A second group of patients who presented late (up to 72 hours of pain), elderly ( $>75$ year of age), with associated medical illnessess such as obstructive airways disease, recent ischaemic heart disease and cerebrovascular disease. Percutaneous abdominal drainage was done with a wide bore Malecoat catheter inserted in the right flank or in both flanks when the abdomen was grosssly distended. All abdominal fluid was drained out. This was also supported by intravenous fluids, gastric aspiration and antibiotics. Oral feeding was started when they improved; the abdomen became softer and bowel sounds had returned. Drainage was discontinued within 7 days. All cases were discharged with proton pump inhibitor for 6 to 12 weeks.

Surgical group: Total of 364 patients were included in this group. After admission they were given intravenous fluid for resuscitation. Surgery done within 6 hours of admission. Repair of the perforation with omental patch enforcement was done in all cases. After thorough lavage with isotonic saline abdominal drainage was instituted. Management policy is summarised in the figure.

\section{Results}

Total 491 duodenal ulcer perforation patients were admitted in our unit. The average age was 43 years ranging from 14-86years. Male: female ratio was $445: 46$. Table 1 shows the clinical characters: all had abdominal pain, 472 had pneumoperitoneum and 52 had hypotension.

Procedure: In the non-surgical group, 56 patients were conservatively treated and 71 patients were offered percutaneous abdominal drainage. Surgical repair with omental patch enforcement done in 364 cases.

Complications: In the surgical group, (table 2) 38 developed chest infection, 19 had different heart ailments like heart failure and myocardial infarction, 26 suffered from wound infections and nine of them had burst abdomen. Eight patients developed pelvic abscess - diagnosis confirmed by ultrasonography, 31 patients had postoperative fever for different reasons eg. like chest infections, subphrenic abscess, pelvic abscess, wound sepsis and urinary tract infections.

In the non-surgical group, 5 patients developed abdominal abscess, 7 had respiratory infections, and heart problems were seen in 2 patients.
Death: In the surgical group total death was 25 $(6.86 \%)$. In the non-surgical group, those who were treated conservatively there was no death, and in the series of percutaneous drainage there were three deaths $(4.2 \%)$ one from cardiac complications, one from respiratory infection and the third developed gastric fistula.

\section{Table 1. Clinical features at presentation}

$\begin{array}{lc}\text { Clinical feanure } & \text { Number } \\ \text { Abdominal pain } & 491 \\ \text { Peritonitis } & 480 \\ \text { Pheumoperitoneum } & 472 \\ \text { Nusea/vomiting } & 122 \\ \text { Hypotension and shock } & 52 \\ \text { Fever } & 85 \\ \text { Diarrhoea } & 42\end{array}$

Table 2. Complications observed in the two groups

$\begin{array}{ccc}\text { Complication } & \text { Surgical group } & \text { Non-surgical group } \\ (n=364) & (n=127)\end{array}$

Respiratory infection

38

2

Heart problems (myocardial

infarction, heart failure)

19

2

Wound infections

Burst abdomen

Gastroduodenal fistula

Subphrenic abscess

Pelvic abscess

Diarrhoea

Death

26

9

2

5

8

24

24

\section{Discussion}

There is continuing debate about whether peptic ulcer perforation cases need to be operated on. It has been estimated that about half of the perforations seal by themselves (5), and a prospective trial comparing conservative treatment with surgical treatment in perforated peptic ulcer has shown no advantage of surgical treatment with regard

\section{Diagnosis}

No risk group

(Healthy patients, early cases)
Moderate risk group

Simple suture with peritoneal lavage
High risk group

(Late cases, elderly patients, co-morbid diseases)

Percutaneous abdominal drainage

Figure. Flow chart of the management protocol of peptic ulcer perforation patients 
to morbidity and mortality (6). Possibly in two-thirds of patients conservative treatment can be safely tried (7). When the patient has only little discomfort and is haemodynamically stable, conservative treatment can be tried under careful surveillance of an experienced surgeon. With nasogastric suction, intravenous fluids and electrolytes, appropriate antibiotics and a proton pump inhibitor, patients' symptoms improve within 12 hours (6).

The concept of conservative treatment is very old. From time to time during emergency operation it has been observed that perforations have sealed by fibrinous adhesions to the liver or omentum, and a loose term 'leaking perforation' has been used to include a group in which the leak has ceased (8). We managed the conservative group of patients with satisfactory results.

Another group of patients were classed as high risk group from our previous experience as well the literature $(9,10)$. Elderly patients, late presentations, and patients with co-morbid conditions are in this category. Mortality rate of this group has ranged from 13 to $30 \%(11,12)$. We offered them percutaneous abdominal drainage followed by conservative treatment. The mortality in this group was remarkably low at $4.2 \%$.

In 1970 s and 1980 s there was a tendency among surgeons to undertake a definitive procedure such as vagotomy and gastrojejunostomy, or vagotomy and pyloroplasty (13). But after the introduction of $\mathrm{H}_{2}$ receptor antagonist the balance is very much in favour of conservative treatment. Some authors have suggested that conservative surgery (repair), peritoneal lavage and antiulcer treatment is the treatment of choice for all patients, even for those with features of chronic ulceration. Fewer patients need elective surgery $(14,15,16)$. The question reopened after the introduction of laparoscopic surgery (17). In our series, we repaired all cases in this group with good peritoneal lavage with a satisfactory outcome.

Outcome in terms of complication rates and death improved in comparison to our previous series (18). Case selection for operative treatment was the key to reducing the mortality rate, which fell to $6.8 \%$, which is comparable to many other series $(14,16,19)$. Wound infection, burst abdomen and chest infection occurred in $7.9 \%, 10.4 \%, 2.4 \%$ respectively, which is much lower than our previous series, and comparable to other series $(20,21)$.

\section{Conclusion}

Complications of peptic ulcer such as perforation still persist, and will possibly continue. For better outcome, case selection for different methods of treatment will reduce the morbidity and mortality. Even adverse situations and patients with very poor general condition can be safely managed. Simple closure followed by drug therapy remains the treatment of choice for a majority of the patients.

\section{References}

1. Svanes C, Salvesen H, Stangeland L, Svanes K, Soreide O. Perforated peptic ulcer over 56 years. Time trends in patients and disease characteristics. Gut 1993; 34: 1666-71.
2. MacKay C. Perforated peptic ulcer in the west of Scotland. A survey of 5343 cases during 1954-63. British Medical Journal 1966; 1: 705-5.

3. Zittel TT, Jehle EC, Becker HD. Surgical management of peptic ulcer disease today - indications, techniques and outcome. Archives of Surgery 2000; 385: 84-90.

4. Jamieson GG. Current status of indications of surgery in peptic ulcer disease. World Journal of Surgery 2000; 24: 256-58.

5. Donovan AJ, Berne TV, Donovan JA. Perforated duodenal ulcer. An alternative therapeutic plan. Archives of Surgery 1998; 133: 1166-71.

6. Crofts TJ, Kenneth GM, Park MB, Stelle RJC, Chung SSC, Li AKC. A randomized trial of non-operative treatment for perforated duodenal ulcer. New England Journal of Medicine 1989; 320: 970-3.

7. Marshall C, Ramaswamy P, Bergin FC, Rosenberg IL, Leaper DJ. Evaluation of a protocol for the non-operative management of perforated peptic ulcer. British Journal of Surgery 1996; 86: 131-34.

8. Taylor H. Aspiration treatment of perforated peptic ulcers Lancet 1951; 6: 7-12.

9. Boey J, Choi SKY, Alagaratnam TT, Poon A. Risk stratification in perforated duodenal ulcers. Annals of Surgery 1987; 105: 22-6.

10. Bodner B, Martin EH, Kim I. Multifactorial analysis of mortality and morbidity in perforated peptic ulcer disease. Surgery, Gynaecology and Obstetrics 1990; 171: 315-22.

11. Irvin TT. Mortality and perforated peptic ulcer: a case for risk stratification in elderly patients. British Journal of Surgery 1989; 76: 215-8.

12. Hermansson M, Stael C, Holsten V, Zilling T. Peptic ulcer perforation before and after the introduction of $\mathrm{H}_{2}$-receptor blockers and proton pump inhibitors. Scandinavian Journal of Gastroenterology 1997; 32: 523-29.

13. Raimes SA, Devlin HB. Perforated duodenal ulcer. British Journal of Surgery 1986; 74: 81-2

14. Svanes C. Trends in perforated peptic ulcer: Incidence, etiology, treatment and prognosis. World Journal of Surgery 2000; 24: $277-83$

15. Bornman PC, Theodorous NA, Jeffery PC. Simple closure of perforated duodenal ulcer, a prospective evaluation of a conservative management policy. British Journal of Surgery 1990; $77:$ 382-5.

16. Towfigh S, Chandler C, Hines OJ, Mcfadden DW. Outcomes from peptic ulcer surgery have not benefited from advances in medical therapy. American Journal of Surgery 2002; 68: 385-9.

17. Windsor JA. The management of perforated duodenal ulcer. New Zealand Medical Journal 1995; 108: 994-5.

18. Rahmn MM, Hossain S. Gastroduodenal perforation of peptic ulcer: review of pattern of surgical procedures in 479 cases. Bangladesh Medical Journal (Khulna) 1995; 27: 15-8.

19. Janet Fy, Leung KL, Lai BSP, Man SS, Dexter S. Predicting mortality and morbidity of patients operated on for perforated peptic ulcers. Archives of Surgery 2001; 136: 90-2.

20. Ball ABS, Thomas PA, Evans SJ. Operative mortality after perforated peptic ulcer. British Joumal of Surgery 1989; 766: $521-2$.

21. Schein M, Geclter G, Freinkel Z, Gerding H. APACH II in emergency operations for perforated ulcers. American Journal of Surgery 1990; 159: 309-13. 